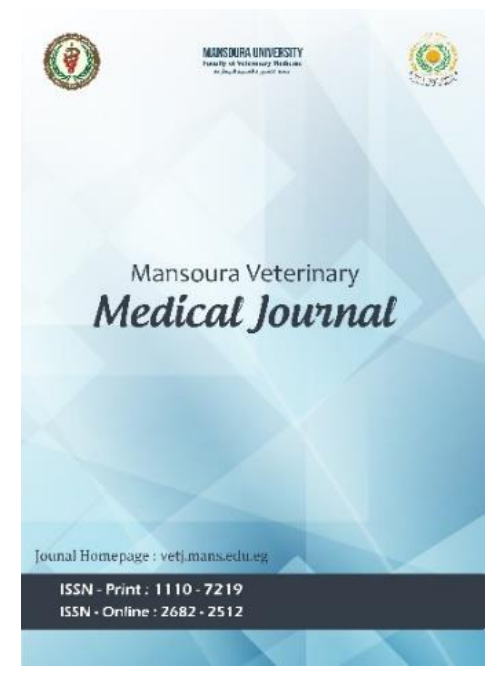

\title{
Prenatal Development of Incisors in the Egyptian Buffalo (Bos bubalis)
}

Mohamed Mostafa, Mesbah El-Sayed, Salah Farag, Galal Youssef

To cite this article: Mohamed Mostafa, Mesbah El-Sayed, Salah Farag, Galal Youssef. Prenatal Development of Incisors in the Egyptian Buffalo (Bos bubalis). Mansoura Veterinary Medical Journal 2020; 21, 3: 80-84.

To link to this article: $\mathrm{https}: / /$ doi.org/10.35943/mvmj.2020.21.314

Published online: 29 September 2020

Submit your article to this journal

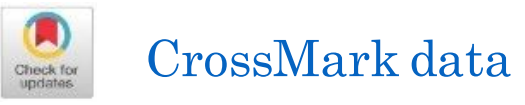




\section{Prenatal Development of Incisors in the Egyptian Buffalo (Bos bubalis)}

Mohamed Mostafa, Mesbah El-Sayed, Salah Farag, Galal Youssef

Department of Anatomy and Embryology. Faculty of Veterinary Medicine Mansoura University, Mansoura, Egypt.

\section{ARTICLE HISTORY}

Received: 13.02 .2020

Revised: 19.05 .2020

Accepted: 20.05 .2020

Address correspondence to Mohamed MMostafa; Tel: +20 01060971550; E-mail: mohamed0506588689@gmail.com

\section{ABSTRACT}

\begin{abstract}
Objective: The teeth play important roles in food mastication, prehension and defense against predators. Although several studies demonstrated the development of teeth in different mammalian species, no data are, to our knowledge, available in Egyptia $n$ buffalos. Therefore, the present investigation was conducted to study the develop ment of incisor teeth in buffaloe.

Design: Descriptive study.

Animals: seventeen buffalo embryos and fetuses of both sexes were used. Their crown vertebral rump lengths (CVRL) ranged from 2 to $46 \mathrm{~cm}$ (equivalent to $38-177$ days old). Procedure: Embryos and fetuses were fixed in $10 \%$ neutral buffered formalin and decalcified by $14 \%$ EDTA solution for several weeks. The samples were dehydrated, cleared and embedded in paraffin wax using standard techniques. Sections were cut on Leitz microtome and mounted on uncoated slides. For general histological structure, a selection of slides was routinely stained with haematoxylin-eosin and examined by the light microscope.

Result: The present study reported for the first time that the prenatal development of incisors in buffalo passes through three sequential stages: the bud, cap and bell stages. Although each stage was easily differentiated based on its form and its distinct developmental features, the three stages were overlapped with each other's. Generally, the bud stage was observed in $11 \mathrm{~cm}$ CVRL buffalo fetuses, meanwhile the cap and bell stages were detected in $23 \mathrm{~cm}$ and $30 \mathrm{~cm}$ CVRL fetuses respectively.

Conclusion and clinical relevance: These results can be used as inductive index for determination of the ages of buffalo embryos and fetuses.
\end{abstract}

Keywords: Buffalo, Incisors, Morphogenesis, Teeth.

\section{INTRODUCTION}

The teeth are white to yellowish-white structures implanted in the bony alveoli of the jaws. They represent about $20 \%$ of the surface a rea of the mouth and playimportant roles in food mastication, prehension and defense against predators [1-4].

Based on their time of appearance during development, the teeth of domestic mammals are referred to either deciduous or permanent teeth. The deciduous teeth are tempora ry structures and a ppear during early life. Thes e teeth are replaced later in life by the permanent teeth. Teeth are also ca tegorized according to their form and position within the oral ca vity into incisors, canines, premolars and molars. The incisors are chisel-shaped teeth located in the frontal part of the dental arch. The premolars and the molars have flat surfaces and constitute the sides of the dental arch. The canines have pointed surface and, when present, are interposed between the incisors and the premolars $[\mathbf{1 , 5 , 6 ]}$. All types of teeth share a basic histological structure consisting of enamel, dentin and cementum [7-9].
The incisor dental formula differs among domestic a nimals. In horse, pig, dog a nd cat, the formulae are: $2\left(\mathrm{Di} \frac{3}{3}\right)=$ 12 and $2\left(1 \frac{3}{3}\right)=12$, while in ox, sheep and goat are $: 2\left(\mathrm{Di} \frac{0}{4}\right)=$ 8and $2\left(1 \frac{0^{3}}{4}\right)=8$, where $\mathrm{Di}$ is deciduous incisors and $\mathrm{I}$ is permanent incisors [6].

Tooth development or odontogenesis is widely agreed to occur in three sequential stages; the bud, cap and bell stages depending on the form of the epithelial enamel organ, which is a portion of the growing tooth [10-12]. Although, the odontogenesis were studied in different mammalian species including sheep [13],equine [14], rabbit [15], guinea pig [16], rat [17], mouse [18, 19] and human [20], the information about the teeth development in Egyptian buffalos is yet preliminary a nd inadequate. Therefore, this study was carried out to shed light on the development of incisor teeth in Egyptian buffalos.

\section{MATERIAL AND METHODS \\ 2.1. Animals}

The pres ent study was carried out on 17 buffalo embryos and fetus es of both sexes. Their crown vertebral rump lengths 
(CVRL) ranged from 2 to $46 \mathrm{~cm}$ (= 38-177.5daysold). These embryos and fetuses were collected from El-Mansoura sla ughterhouse immediately after evisceration and from the aborted and the premature-birth fetuses on the veterinary field by personal communication. The ages of the collected buffalo embryos and fetuses were es timated by the formula given by Abd El-Raouf et al. (1968) [21]. The details of samples us ed in the present studya re summarized in table 1.

\subsection{Histological analysis}

Embryos and fetuses were fixed in 10\% neutral buffered formalin. The specimens were decalcified in $14 \%$ EDTA solution for several weeks. The samples were dehydrated, cleared and embedded in paraffin wax using standard techniques. $5 \mu \mathrm{m}$ thickness sections were cut on Leit mi crotome, mounted on uncoated slides, and stained with Ehrlich's Heamatoxylin and Eosin. All tissue processing and staining were adopted according to Suvarna et al. (2018) [22] The prepared specimens were examined by the light microscope and representative images were captured using digital camera.

\subsection{Nomenclature}

The nomenclature used in the present study were adopted according to Nomina Anatomica Veterineria (2012) [23], Nomina Histologica Veterineria (2018) [24] and the available literatures.

Table 1. Embryos and fetuses used in the present study

$\begin{array}{lll}\text { Serial number } & \text { CVRL (cm) } & \text { Age (days) } \\ 1 & 2 & 38 \\ 2 & 6 & 56 \\ 3 & 10 & 74 \\ 4 & 11 & 78 \\ 5 & 12 & 83 \\ 6 & 14 & 92 \\ 7 & 16 & 101 \\ 8 & 18 & 110 \\ 9 & 19 & 114 \\ 10 & 21 & 121 \\ 11 & 23 & 125 \\ 12 & 26 & 132 \\ 13 & 30 & 141 \\ 14 & 32 & 146 \\ 15 & 39 & 161 \\ 16 & 43 & 170 \\ 17 & 46 & 177 \\ & & \end{array}$

\section{RESULTS}

In $2 \mathrm{~cm}$ CVRL buffalo embryos, primordia of the incisors appeared as thickened areas at the primary epithelial band. These a reas were mi croscopically formed of several layers of undifferentiated epithelial cells and repres ented the first sign of odontogenesis in buffalo (Figure 1. A).

In $11 \mathrm{~cm}$ CVRL buffalo fetuses, the teeth development appeared more progressed compared to $2 \mathrm{~cm}$ CVRL embryos.
The distal end of dental lamina seemed wider and more flattened to form the tooth bud. Such bud composed of large number of condensed epithelial cells and was easily demarcated from the larger and less condensed vestibular lamina (Figure 1. B).

In $21 \mathrm{~cm}$ CVRL buffalo fetuses, the developing tooth achieved dramatic changes and a ppeared in cap stage. The cap-shaped tooth was formed of an enamel organ and underlying dense mesenchyme. The enamel organ was differentiated into three layers: the outer enamel epithelium, the inner enamel epithelium and the stellate reticulum. The outer and inner enamel epithelia was composed of cuboidal to col umnar epithelium. The stellate reticulum was consisted mainly of polygonal lightly stained epithelial cells. The tooth mesenchyme appeared condensed and was seen invading the concave free end of the enamel organ to form the future dental papilla. The rest of surrounding mesenchyme will form the presumptive dental follicle. It is noteworthy that, at this stage of development the permanent tooth bud was also detected evaginating from the dental lamina near the attached end of the enamel organ (Figure 1. C).

In $30 \mathrm{~cm}$ CVRL buffalo fetuses, the developing tooth a ppea red in the early bell stage. The dental lamina a ppeared completely disintegrated. The mesenchyme of the dental papilla became denser and the stellate reticulum of the ena mel orga nappeared va cuolated giving the typical stellate appearance of this part. The outer enamel epi thelium was cuboidal while the inner enamel epithelium was columnar (Figure 1. D, E).

In $39 \mathrm{~cm}$ CVRL buffalo fetuses, the tooth achieved more growth and reached the late bell stage. The dental papilla was elongated, pointed and came in contact with the outer enamel epithelium and appeared more vascularized and transformed into tooth pulp. The inner enamel epithelium differentiated into the ameloblasts and was darkly stained possibly due to the immense production of enamel at this stage. The most outer layer of the tooth pulp differentiated into cuboidal or low columnar epithelium to form the odontoblasts. The latter cells a ppea red lightly stained due to dentin secretion (Figure 1. F, G).

In $46 \mathrm{~cm}$ CVRL buffal ofetuses, tooth crown a cquired the final shape where it became more elongated, compressed, and formed about two third of the tooth length. The dentin was also thick being thicker proximally while its thickness decreased downward till fads out at the level of the tooth cervix. Enamels was highly dense and thick at the proximity of the crown and became thin distally. Tooth pulp was narrow along the length of the tooth crown whereas it tapered proximally forming very narrow strip at the crown summit (Figure 1. H). The appearance of the developing buffalo incisors and their mineralization pattern at $46 \mathrm{~cm} \mathrm{CVRL}$ is shown in Figure 1.I.

\section{DISCUSSION}

The present study revealed that, the teeth development of the growing buffalo fetuses followed a certain pattern of 
sequential developmental changes where their growth occurs in three subsequent stages: the bud, cap and bell stages depending on to the form of the epithelial enamel organ. This observation is coincided with statement of Catón et al. (2009) in mouse fetuses [10].
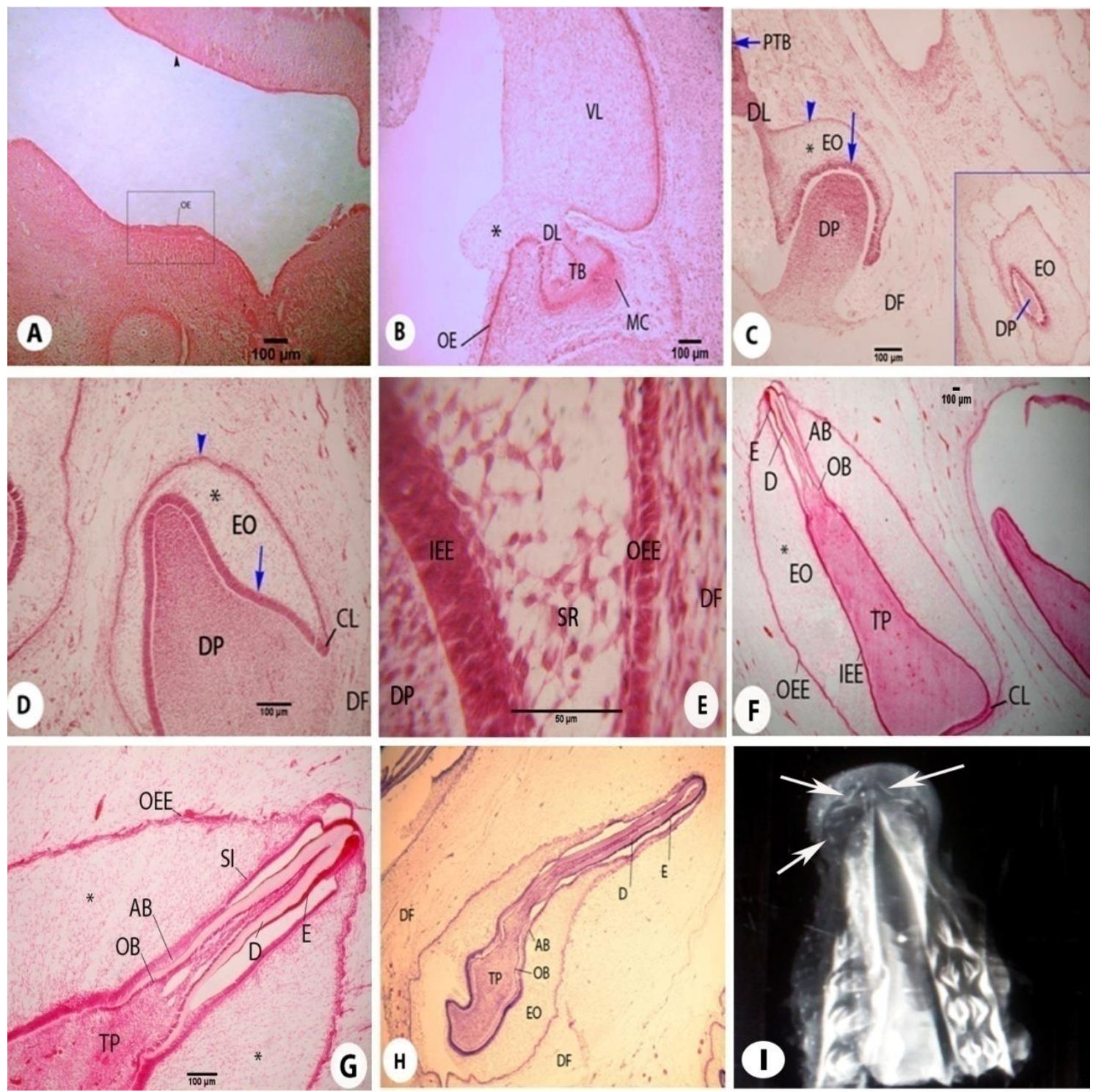

Figure 1. Different stages of incisor teeth development in buffalo. A- Light photomicrograph of buffalo embryo head $2 \mathrm{~cm}$ CVRL, showed the lower jaws exhibiting the oral epithelium (OE) with an epithelial thickening of the primary epithelial band (intersect), note upper jaw (arrowhead). H\&E stain. B- Light photomicrograph of buffalo fetus head $11 \mathrm{~cm} \mathrm{CVRL,} \mathrm{showed} \mathrm{thickened}\left({ }^{*}\right)$ oral epithelium (OE), dental lamina (DL), tooth bud (TB) and vestibular lamina (VL). H\&E stain. C- Light photo micrograph of buffalo fetus head $21 \mathrm{~cm}$ CVRL, showed dental lamina (DL), enamel organ (EO) with an outer enamel epithelium (arrow head), stellate reticulum (asterisk), inner enamel epithelium (arrow), dental papilla (DP), dental follicle (DF) and permanent tooth bud (PTB). Intersect: cross section in enamel organ (EO) enclose the dental papilla (DP). D- Light photomicrograph of buffalo embryo head $30 \mathrm{~cm} \mathrm{CVRL,} \mathrm{showed} \mathrm{the} \mathrm{outer} \mathrm{layer} \mathrm{of} \mathrm{enamel} \mathrm{organ} \mathrm{(arrowhead),}$ stellate reticulum (asterisk), inner enamel epithelium (arrow), dental papilla (DP), cervical loop (CL) and dental follicle (DF). E- Higher magnification of (D) showed, outer layer of enamel organ (OEE) composed of cuboidal epithelium, highly vacuolated cells of stellate reticulum (SR), inner enamel epithelium (IEE) composed of columnar epithelium with nuclei at two levels and dental papilla (DP). F- Light photomicrograph of buffalo fetus head $39 \mathrm{~cm}$ CVRL, showed tooth pulp (TP) appeared more pointed and contact the outer enamel epithelium (OEE). The inner enamel epithelium differentiated into ameloblast (AB), which secret enamel (E), the most outer layer of tooth pulp differentiated into odontoblast cells (OD) which secret dentin (D), stellate reticulum (asterisk), cervical loop (CL), outer enamel epithelium (OEE) and inner enamel epithelium (IEE). G- Higher magnification of (F), the developing tooth showed tooth pulp (TP), odontoblast (OD), dentin (D), ameloblast cells (AB), enamel (E), stellate reticulum (asterisk) and stratum intermedium (SI). $\mathrm{H}$ - Light photomicrograph of buffalo fetus head $46 \mathrm{~cm}$ CVRL, showed tooth pulp (TP) appeared more elongated and narrower, ameloblast (AB) and odontoblast (OB) close to each other distally, and separated by enamel (E) and dentin (D) proximally, note enamel organ (EO) and dental follicle (DF). I- X-ray photograph of $46 \mathrm{~cm}$ CVRL buffalo fetus head showing the mineralization of the developing incisors is evident at this stage of development (arrows).

The $2 \mathrm{~cm}$ CVRL (about 38 days-old) buffalo embryo exhibited thickened areas at certain parts of oral epithelium known as primary epithelial band, the first signs of odontogenesis. Such findings were also reported by previous studies in 33 days-old sheep fetuses [13]; 15 days-old rabbit fetuses [15]; 13 days-old rat fetuses [15]; 22 days-old guinea 
pig fetuses [16];11-11.5 days mouse fetus es [18, 26]; and 37 days old human fetuses [2]. Furthermore, in 6 weeks-human fetuses the band of thickened oral epithelium was horseshoeshaped [33].On other hand, in mouses fetuses the first indication of tooth development was a condensation of mes enchymal tissue beneath the dental epithelium [34].

The present study revealed that the dental lamina became more developed, enlarged and deepened in the underlying mesenchymal tissue forming the tooth bud in 11 cm CVRL (a bout 78.5 days-old) buffalo fetus in accordance with that described by previous investigators in 35 days-old sheep [13]; in 25-30 days-old guinea pig fetuses [16]; in17 days-old rat fetuses [17] and in 13 days-old mouse fetuses [27]. However, the tooth bud of molar teeth in the albino mouse was described as an club-shaped bulge [29].

In 21cm CVRL (about 121.5 days-old) buffalo fetus, dramatic devel opmental changes were prominent whereas the incisor tooth beca me in true cap stage. The tooth cap with the dense underlying mesenchyme was differentiated into enamel organ that was distinguished into three layers, an outer and inner enamel epithelium with cuboidal to columnar epithel ium res pectively. The third layer was stel late reticulum and composed of polygonal lightly stained epithelium. These findings were supported by previous reports in 15 days-old rabbit fetuses [15]; rat fetuses [17] and human fetuses [31]. Moreover, in 15 days-old rabbit fetuses, the bud-shaped tooth germ has enlarged very greatly in relation to the rest of the lamina and became as an inverted saucer [15].

In $30 \mathrm{~cm}$ CVRL (a bout 141.5 days-old) buffalo fetus, the dental lamina was completely disappeared however, dental papillae mesenchyme appeared more dense Moreover, the stellate reticulum cells of enamel organ a ppeared vacuolated and their nuclei lodged in narrow strip of cytoplasm giving the typical stellate appearance of the cells. The outer enamel epithelium was cuboidal while the inner enamel epithelium was columnar and their nuclei appeared in two levels.

In $39 \mathrm{~cm}$ CVRL (a bout 161.75 days-old) buffalo fetus, the inner enamel epithelium became more columnar and differentiated into ameloblast which secrets the tooth enamel that a ppeared darkly stained. The most outer layer of the tooth pulp differentiated into cuboidal or low columnar epithelium and called odontoblast and secret dentin which appeared more lightly stained. These findings a re concurrent to that observed in mouse fetuses [35].

In $46 \mathrm{~cm}$ CVRL (about 177.5 days-old) buffalo fetus, the odontoblast appeared low columnar with basally located nuclei. These cells secret dentin earlier than enamel which differentiated into lighter strip close to odontoblast called predentin and outer dark strip is the dentin. The a meloblast appeared more elongated than odontoblast and secrets enamel. Such condition was correl ated with that mentioned by other inves tigators in fetuses of other species including horse [14]; mouse [27] and human [25]. In addition, Tucker et al. (2004) in mouse fetuses [27] added that cytodifferentiation ta ke places within the bell phase of tooth proliferation. on the contrary, Huang et al. (2010) in mouse fetuses [32] reported that the a mel oblasts and odontoblasts start to distinguish after the cap shape is produced. Our findings in $46 \mathrm{~cm}$ CVRL buffalo fetus also revealed that the tooth pulp became narrower and highly elongated and pointed extending from the future incisal surface to the future root of the tooth. The dentin was thicker proximally and extend distally to cover about three quarter of the developing tooth. Tooth enamel became more pronounced and relatively thick and extends more distally. Stellate reticulum was formed of very na rrow strip at the middle and nearly disappeared proximally, and still observed and huge distally. The tooth follicle was more clear, distinguishable and appeared fibrous

\section{Conclusion}

The present study reported for the first time that the prenatal development of incisors in buffalo passes through three sequential stages: the bud, cap and bell stages. Although each stage was easily differentiated based on its form and its distinct developmental features; the three stages are overlapped with each other's. The bud stage was observed in $11 \mathrm{~cm}$ CVRL buffalo fetuses, meanwhile the cap and bell stages were detected in $23 \mathrm{~cm}$ and $30 \mathrm{~cm} \mathrm{CVRL}$ fetuses respectively. Further studies are needed to study the mol ecular mechanisms controlling the devel opment of teeth in buffalo.

\section{Conflict of interest statement}

The authors declare that there is no a ny conflict of interest in the current res earch work

\section{Animal ethics committee permission}

The current research work is permitted to be executed according to standards of a nimal research committee in Faculty of Veterinary Medicine, Mansoura University.

\section{Authors' contributions}

Mohamed Mostafa Mohamed Mansour conducted the experiment and drafted the manuscript; Salah El-Morsy Farag, Mesbah Abd El-Gawwad El-Sayed, and Galal Ahmed ElSayed Youssef designed the experiments and contributed to the final version of the manuscript.

\section{REFERENCES}

[1] Sisson, Septimus, Grossman, James Daniels. The Anatomy of the Domestic Animals. 3rd edition, Saunders, Philadelphia.1953.

[2] Antonio Nanci: Ten Cates Oral Histology Development Structure and Function. $8^{\text {th }}$ edition, E. H. Sci, Canada ;70-92, 2015.

[3] Evans, Howard E, De Lahunta, Alexander. Miller's anatomy of the dog-EBook, $3^{\text {rd }}$ edition. E. H. Sci. China, 2013.

[4] Eurell, J. A. and B. L. Frappier. Dellmann's textbook of veterinary histology. $9^{\text {th }}$ edition, John Wiley \& Sons, canada, 2013.

[5] Liebich, Hans-Georged. Veterinary anatomy of domestic mammals textbook and color atlas. $2^{\text {nd }}$ edition, Schattauer, Germany, 2004.

[6] Lahunta, A de Habel, Robert E. Applied veterinary anatomy. $2^{\text {nd }}$ edition, WB Saunders, Philadelphia, PA, 1986.

[7] Banks, William J. Applied veterinary histology, $3^{\text {rd }}$ edition, Mosby-Year Book, Inc, USA, 1993. 
[8] McGeady, Thomas A, Quinn, Peter J, Fitz Patrick, ES, Ryan, MT, Kilroy, David, Lonergan, Pat. Veterinary embryology, 1st edition, John Wiley \& Sons.UK, 2006.

[9] Hyttel, Poul, Sinowatz, Fred, Vejlsted, Morten, Betteridge, Keith. Essentials of domestic animal embryology, 1st edition, E. H. Ss, china. 2010.

[10] Koyama, Tomoichiro, Iseki, Sachiko, Tsutomu, Hidefumi, Hayashi, Yoshio, Leatherman, Judith L, Golden, Eleanor B, Noji, Sumihare. Polarizingactivity, Sonic hedgehog, tooth development in embryonic and postnatal mouse. Developmental dynamics: an official publication of the A. A . 206: PP: 59-72, 1996.

[11]Catón, Javier, Tucker, Abigail S. Current knowledge of tooth development: patterning andmineralization of the murine dentition. J. A.; 214: 502-515, 2009. https://doi.org/10.1111/j.14697580.2008.01014.x

[12] Jernvall, Jukka, Thesleff, Irma . Tooth shape formation and tooth renewal: evolving with the same signals. Development.;139: 3487-3497, 2012. https://doi.org/10.1242/dev. 085084

[13] Witter, K, Mišek, I. Time programme of the early tooth development in the domestic sheep (Ovisaries, Ruminantia). Acta. Vet . Brno 68: PP: 38, 1999. https://doi.org/10.2754/avb199968010003

[14] Kirstie Dacre, B., MSc, Cert EM Int Med PhD. Applied Equine Dental Development. Kirstie Dacre, B., MSc, Cert EM Int Med PhD..AAEP, USA, 2006.

[15] Glasstone, Samuel. A comparative study of the development in vivo and in vitro of rat and rabbit molars. Proceedings of the Royal Society of London. $\quad$ Series B-B.S.; 126: 315-330, 1938. https://doi.org/10.1098/rspb.1938.0059

[16] Harman, Mary T Smith, Arlene. Some observations on the development of the teeth of Cavia cobaya. The Anatomical Record.66: 97-111. 1936 https://doi.org/10.1002/ar.1090660110

[17] Lefkowitz, William, Bodecker, Charles F, Mardfin, Dorothy F. Odontogenesis of the rat molar: prenatal stage. J Dent Res..32: 749-772, 1953. https://doi.org/10.1177/00220345530320060301

[18] Mitsiadis, Thimios A, Chéraud, Yvonnick, Sharpe, Paul, Fontaine-Pérus, Josiane. Development of teeth in chick embryoafter mouse neural crest transplantations. Proceedings of the N. A. S.100:PP 6541-6545, 2003. https://doi.org/10.1073/pnas.1137104100

[19] Li, Chun-Ying, Prochazka, Jan, Goodwin, Alice F, Klein, Ophir D. Fibroblast growth factor signaling in mammalian tooth development. Odontology. 102: PP:1-13,2014. https://doi.org/10.1007/s10266-0130142-1

[20] Chinsembu, Kazhila C. Teeth are bones: Signature genes and molecules that underwrite odontogenesis. J. Med. Gen \& G.; 4:13-24, 2012. https://doi.org/10.5897/JMGG11.022

[21] Abd El-Raouf, M. and El-Naggar, M. A.: Biometry of the Egyptian buffalo fetus. J. Vet. Sci; U. A. R. 5: 37-43, 1968.

[22] Suvarna, Kim S, Layton, Christopher, Bancroft, John D. Bancroft's Theory and Practice of Histological Techniques E-Book,7th edition, E. H. Sci. china, 2018.

[23] Waibl, H., Gasse, H., Constantinescu, G. M., Hashimoto, Y., \&Simoens, P. N.A.V. The international committes on vet. Gross. Anat. Nom. The Editorial Committee Hannover, Sapporo, Japan.2012.

[24] N.H.V. International committee on veterinary Histological Nomenclature (ICVHN). General assemble of the world association of veterinary anatomists. 2018.

[25] James K. Avery, Daniel J. Chiego. Essentials of Oral Histology and Embryology, 8th edition .E. H. Sci. china . 2008.

[26] Golonzhka, O,et al. Ctip2/Bcl11b controls ameloblast formation during mammalian odontogenesis. Proceedings of the N. A. S .106: 4278-4283, 2009. https://doi.org/10.1073/pnas. 0900568106

[27] Tucker, A. and P. Sharpe . The cutting-edge of mammalian development; how the embryo makes teeth. N. R. Gs .5: 499, 2004. https://doi. org/10.1038/nrg1380

[28] Sharpe, Paul T, Young, Conan S.Test-tube teeth. Sci. A.; 293: 34-41, 2005. https://doi.org/10.1038/scientificamerican0805-34[29] Cohn, Sidney A. Development of the molar teeth in the albino mouse. A. J.Anat.101: 295319, 1957. https://doi.org/10.1002/aja.1001010205

[30] Symons, N. Alkaline phosphatase activity in the developing teeth of the rat." J. Anat. 89: 238, 1955.

[31] Ferraro, James W, Hakola, S. Fundamentals of maxillofacial surgery human, 6th edition Springer Science \& Business Media.USA. 2012.
[32] Huang, Xiao Feng, Chai, Yang TGF- $\beta$ signalling and tooth development. Chin J Dent Res. 13:7-13, 2010.

[33] Young, Barbara, Woodford, Phillip, O'Dowd, GeraldineWheater's . Functional Histology E-Book: A Text and Colour Atlas, E. H.Sci, 2013.

[35] Mitsiadis, T. A. and D. Graf. Cell fate determination during tooth development and regeneration." Birth Defects Research Part C: Embryo Today: Reviews.; 87:199-211, 2009. https://doi.org/10.1002/bdrc. 20160 